LAWRENCE LIVERMORE N A T IO N A L LABORATORY
Phase Effects on Mesoscale Object X-ray Absorption Images

H. E. Martz, Jr., M. B. Aufderheide, A. Barty, S. K. Lehman, B. J. Kozioziemski, D. J. Schneberk

October 18, 2004 
This document was prepared as an account of work sponsored by an agency of the United States Government. Neither the United States Government nor the University of California nor any of their employees, makes any warranty, express or implied, or assumes any legal liability or responsibility for the accuracy, completeness, or usefulness of any information, apparatus, product, or process disclosed, or represents that its use would not infringe privately owned rights. Reference herein to any specific commercial product, process, or service by trade name, trademark, manufacturer, or otherwise, does not necessarily constitute or imply its endorsement, recommendation, or favoring by the United States Government or the University of California. The views and opinions of authors expressed herein do not necessarily state or reflect those of the United States Government or the University of California, and shall not be used for advertising or product endorsement purposes.

This work was performed under the auspices of the U.S. Department of Energy by University of California, Lawrence Livermore National Laboratory under Contract W-7405-Eng-48. 


\title{
Phase Effects on Mesoscale Object X-ray Absorption Images ${ }^{1}$
}

\author{
H. E. Martz, Jr., M. B. Aufderheide, A. Barty, S. K. Lehman, \\ B. J. Kozioziemski, and D. J. Schneberk \\ UCRL-TR-207286, Lawrence Livermore National Laboratory, Livermore, CA 94550
}

\section{Project overview}

At Lawrence Livermore National Laboratory particular emphasis is being placed on the nondestructive characterization (NDC) of 'mesoscale' objects.[Martz and Albrecht 2003] We define mesoscale objects as objects that have mm extent with $\mu \mathrm{m}$ features. Here we confine our discussions to $\mathrm{x}$-ray imaging methods applicable to mesoscale object characterization.

\section{Project goals}

The goal is object recovery algorithms including phase to enable emerging high-spatial resolution xray imaging methods to "see" inside or image mesoscale-size materials and objects. To be successful our imaging characterization effort must be able to recover the object function to one micrometer or better spatial resolution over a few millimeters field-of-view with very high contrast.

\section{Relevance to the lab Mission}

Specific LLNL programs that would benefit from this new capability include development of novel sensors for NAI applications, study of explosive samples for DOD and DOE, and high energy density physics, and inertial confinement fusion experiments for the National Ignition Facility (NIF).

\section{FY04 Accomplishments and Results}

Our approach includes the research, development and validation of algorithms to model phasecontrast effects observed in x-ray systems, and to use these algorithms for quantitative object recovery. This requires three tasks. First, we are modifying HADES [Aufderheide, et al. 2000] to model phase contrast for point projection imaging and investigating whether multislice techniques within the object are needed to fully capture the physics seen in phase-contrast data. Secondly, we are developing several object recovery approaches using parameter-based [Chambers, et al. 2003] and voxel-based techniques [Barty, et al. 2000]. Thirdly, we are validating these simulations against $\mathrm{x}$-ray systems using well-known objects. At the end of this R\&D, we will have a set of validated xray codes for modeling and reconstructing objects including the effects of phase.

We have extended HADES to be able to calculate both the x-ray attenuation and phase of complex objects, a capability that is already showing relevance to other LLNL projects. We are currently working on incorporating the Fresnel integral into HADES to mix phase and absorption information as required by the diffractive and refractive phenomena.

We have reached the point in x-ray imaging where the spatial resolution and object dimensions could theoretically result in the wave diffraction effects within the sample becoming significant. We studied this possibility numerically using a paraxial approximation wave propagation code with a multislice method. We modeled realistic parts of interest at worst-case $\mathrm{x}$-ray energies, $\sim 8-\mathrm{keV}$. The conclusion, based upon the results of the simulation code, is that diffraction effects on the measured data will be insignificant (Figure 1).

Typically more than one image is required to separate the object phase and absorption structure. However, for the case of an object composed of a single material, both the projected phase and

\footnotetext{
${ }^{1}$ This work was performed under the auspices of the U.S. Department of Energy by the University of California, Lawrence Livermore National Laboratory under Contract No. W-7405-Eng-48.
} 
amplitude through an object are directly related to the projected material thickness. This enables the object to be described in a single variable, the object thickness, as opposed to two variables, amplitude and phase. This in combination with the transport of intensity equation (TIE) yields a strategy for reconstructing the object taking into account phase contrast features in the detected image.[Paganin, et al. 2002] This approach has been implemented numerically, and we are in the process of investigating its application to reconstructions of surrogate objects (Figure 2).

Continued modeling has identified that there is a shift in position of key features within an object that can only be explained by incorporating phase effects into the system modeling (Figure 3). Note how the location of the inner edge when phase effects are included appears to shift position with changing energy, even though part dimensions were held constant. This effect has also been seen in experimental data collected at the APS on surrogate parts, and we are in the process of developing techniques to quantify the dimensional shift introduced.

A key part of this proposed project is the validation of the simulation and object-recovery codes. Two things are required to validate $\mathrm{x}$-ray simulation codes: the x-ray system being modeled and a well-known object (also known as a phantom or reference standard). We have designed a multislice phantom that can be modeled and used to validate the simulations (Figure 4).

\section{FY05 Proposed Work}

We will focus on 2D simulations and uniform object recovery. HADES incorporation of phase effects and the multislice code will be validated using phantoms. Object recovery algorithms will be investigated and validated for uniform single material objects and parametric reconstructions using HADES.

\section{Related References}

Aufderheide, M. B., D. M. Slone, and A. E. Schach von Wittenau (2000), "HADES, A Radiographic Simulation Code," in Review of Progress in Quantitative Nondestructive Evaluation, 20A, AIP Conference Proceedings 557, pp. 507-513.

Aufderheide, M.B., Barty, A., and Martz, H.E. (2004), "Simulation of Phase Effects in Imaging for Mesoscale NDE", Lawrence Livermore National Laboratory, Livermore, CA, UCRL-CONF206263, submitted to Review of Progress in Quantitative Nondestructive Evaluation,.

Barty, A., K.A. Nugent, A. Roberts, and D. Paganin (2000), "Quantitative phase tomography," Opts. Comm. 175, pp. 329-336.

Chambers, D. H., D. M. Goodman, and R. R. Leach (2003), "As-built model generation for a cylindrical test object," Lawrence Livermore National Laboratory, Livermore, CA, UCID-TR200695.

Martz Jr., H. E., and G. F. Albrecht, (2003), "Nondestructive Characterization Technologies for Metrology of Micro/Mesoscale Assemblies," Proceedings of: Machines and Processes for Micro-scale and Meso-scale Fabrication, Metrology, and Assembly, ASPE 2003 Winter Topical Meeting, Gainesville, FL, January 22-23, pp.131-141.

Paganin, D., S. C. Mayo, T. E. Gureyev, P. R. Miller, and S. W. Wilkins (2002), "Simultaneous phase and amplitude extraction from a single defocused image of a homogeneous object," $J$. Micros. 206, pp. 33-40. 
Geometry 3 at $8 k e V$ Measured at $100 \mathrm{~mm}$
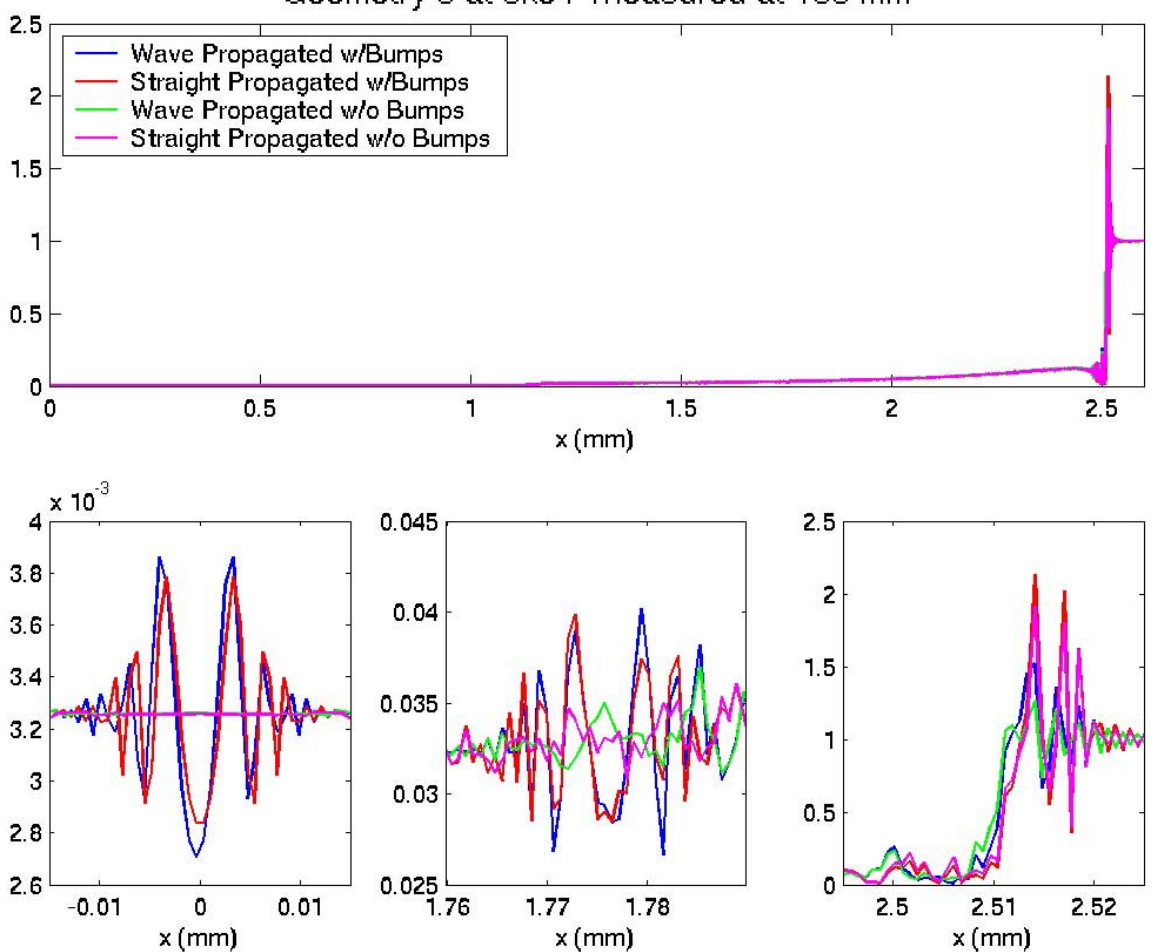

Figure 1 Simulation results from a paraxial approximation wave propagation code using a multislice method and straight ray tracing. The modeled object is a 3.2-mm o.d. double shell capsule with a $\mathrm{CH}$ inner and outer shell. On the outside of the outer shell there are 1-um spherical features or bumps that are shown in detail in the bottom image.

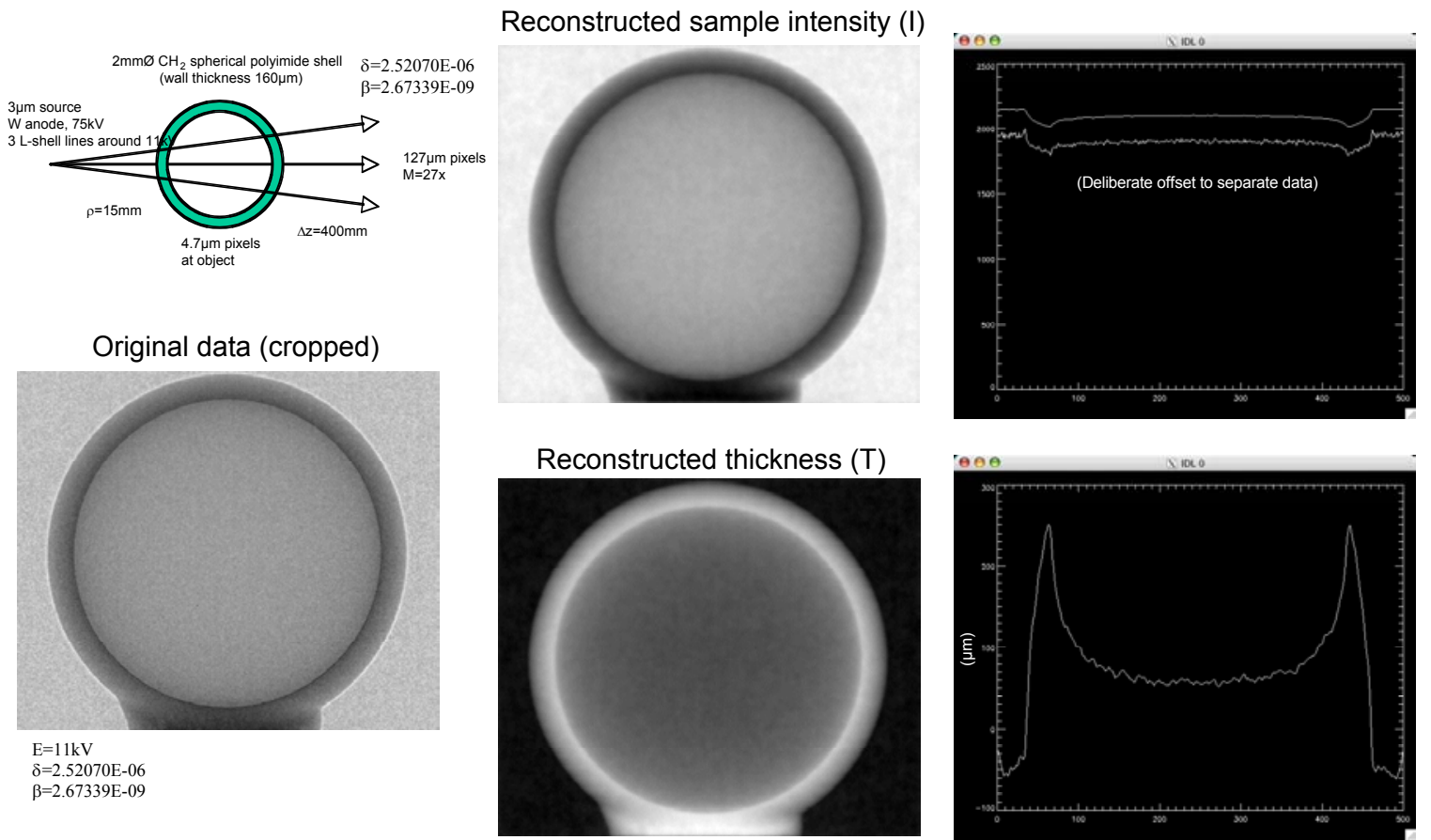

Figure 2: Application of the single-material reconstruction algorithm to LLNL's KCAT data of a polystyrene sphere. This algorithm accounts for the phase contrast features in the data and reconstructs the exit wave of the object. Both the thickness and transmitted intensity are computed, and the bright edges due to phase contrast are removed. This data is ideal for input into tomographic reconstruction algorithms for 3D object reconstruction. 


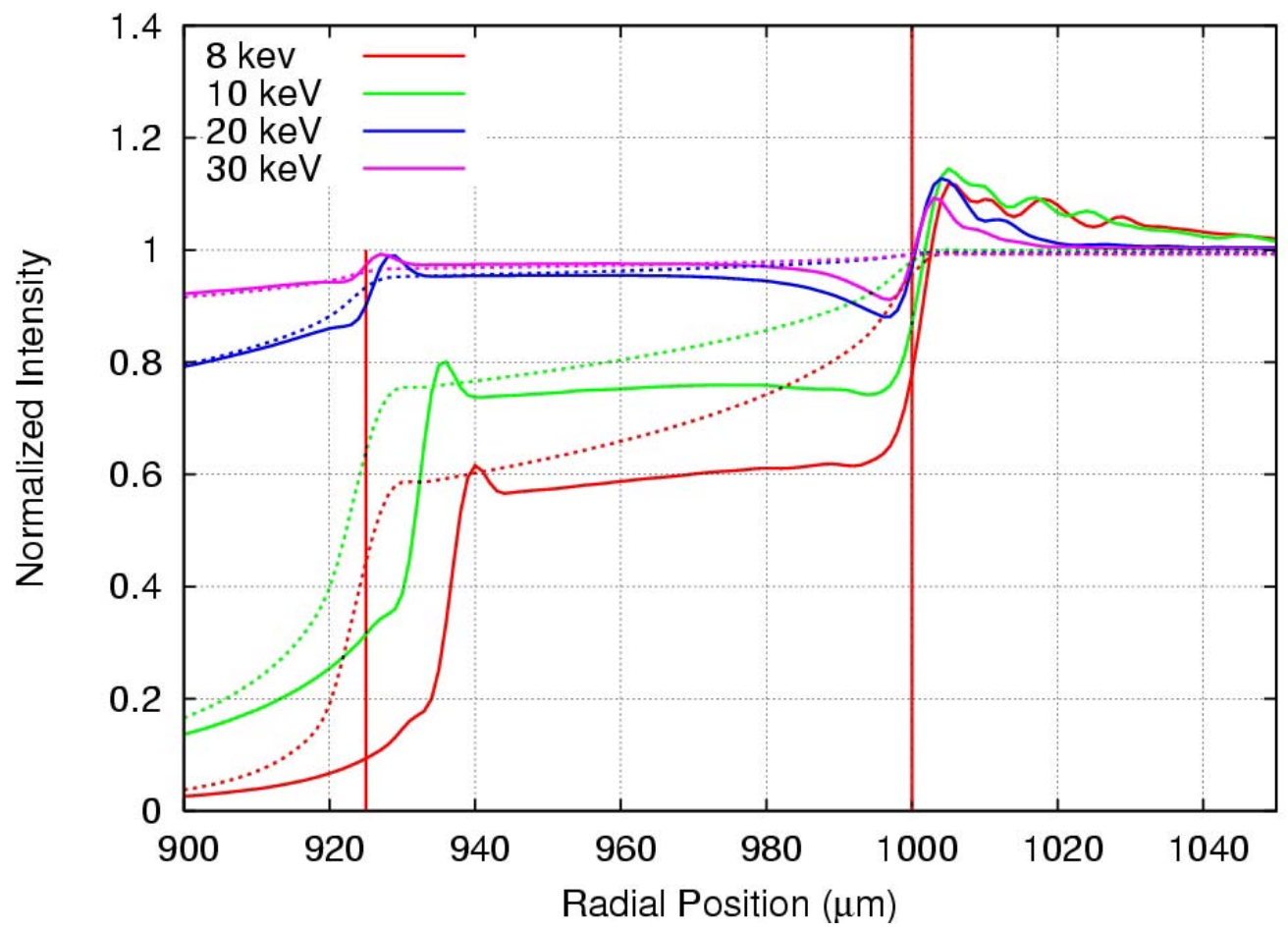

Figure 3 Simulated results for x-ray imaging of a 75-um thick CVD diamond $\left(\rho 3.3 \mathrm{~g} / \mathrm{cm}^{3}\right)$ shell coated on a 925-um radius $\operatorname{SiN}\left(\rho 3.44 \mathrm{~g} / \mathrm{cm}^{3}\right)$ mandrel. The object-to-detector distance is $200 \mathrm{~mm}$. The object pixel pitch is 1 um, convolved with a Gaussian blur with a sigma value of $2.0 \mathrm{um}$. The image still shows the shift in position of the interface with energy (refractive index really). I think that's all the relevant parameters. Results with phase effects included are given by the solid lines. Results with no phase effects are given by the dashed lines. Note the dramatic difference in the phase data as a function of energy (due to the change in refractive index) compared with the no phase results.

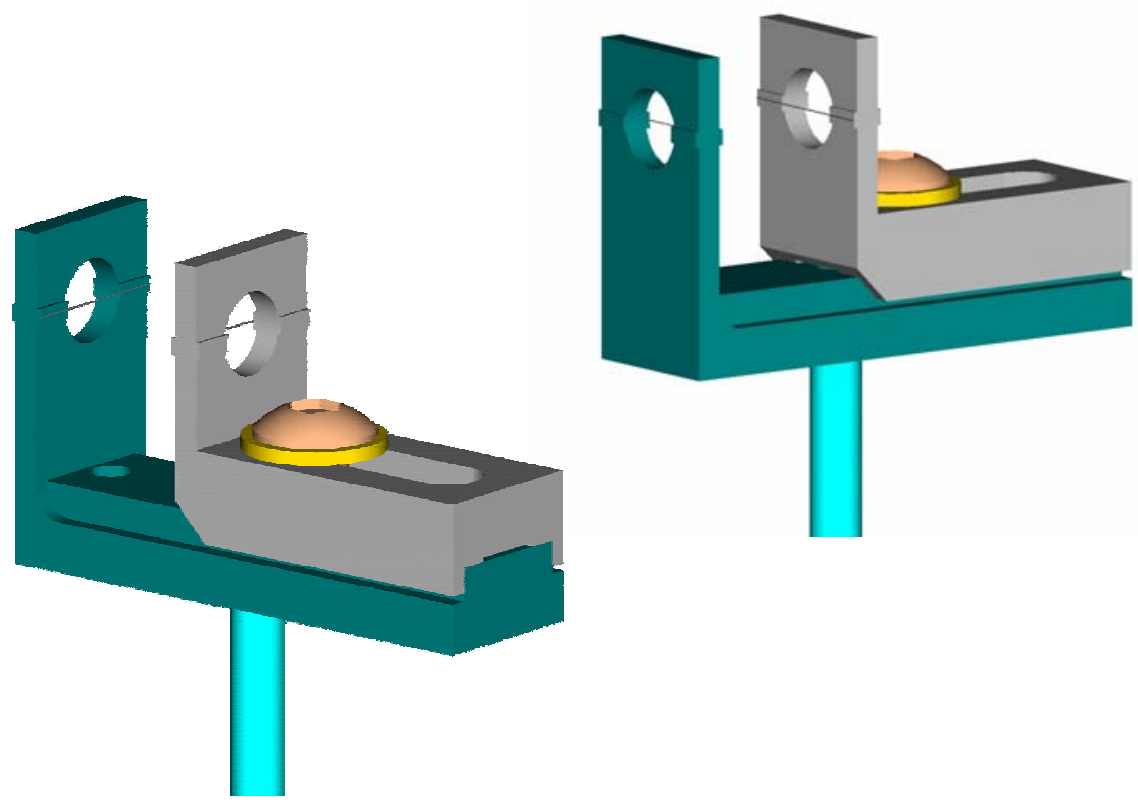

Figure 4 Phantom designed to validate the multislice (also known as the beam propagation) method to solve the paraxial wave equation to simulate $\mathrm{x}$-ray microscopy of mesoscale objects and determine if ray tracing will suffice. The phantom has two carbon filaments of 6- $\mu \mathrm{m}$ diameter that can be placed at different location $(0-10 \mathrm{~mm})$ along the $\mathrm{x}$-ray beam axis. 